Pacific Journal of Mathematics

ISOMETRIC IMMERSIONS OF SPACE FORMS IN SPACE 


\title{
ISOMETRIC IMMERSIONS OF SPACE FORMS IN SPACE FORMS
}

\author{
John Douglas MoORE
}

\begin{abstract}
Let $M$ be a connected $n$-dimensional space form isometrically immersed in a simply connected $(2 n-1)$-dimensional space form of strictly larger curvature. If $M$ is minimal, it is proven that it must be a piece of the flat Clifford torus in the $(2 n-1)$-sphere. If $M$ is complete and simply connected, it is proven that $M$ possesses a global coordinate system whose coordinate vectors are unit-length asymptotic vectors.
\end{abstract}

Introduction. A well-known theorem of David Hilbert states that a complete two-dimensional riemannian manifold of constant negative curvature cannot be isometrically immersed in three-dimensional euclidean space [5], [7, p. 265]. There is reason to believe that the natural generalization of Hilbert's theorem to higher dimensions would be the following conjecture: A complete $n$-dimensional riemannian manifold of constant negative curvature cannot be isometrically immersed in $E^{2 n-1}$. If completeness is strengthened to compactness the conjecture is known to be true by work of Chern, Kuiper, and Otsuki [6, vol. 2, p. 29].

The local problem of isometrically immersing a space form in a space form was studied by Élie Cartan [3]. He used his theory of exterior differential systems to show, among other things, that real analytic $n$-dimensional submanifolds of constant negative curvature in (2n-1)-dimensional euclidean space $E^{2 n-1}$ depend upon $n(n-1)$ functions of a single variable. Cartan also showed that no $n$-dimensional hyperbolic space form can be isometrically immersed in $E^{2 n-2}$. To construct an explicit example, we choose nonzero real numbers $a_{i}, 1 \leqq i \leqq n-1$, so that $\sum_{i} a_{i}{ }^{2}=1$, and we define an immersion from

$$
D=\left\{\left(y_{1}, y_{2}, \cdots, y_{n}\right) \in \boldsymbol{R}^{n} \mid y_{n}<0\right\}
$$

into $E^{2 n-1}$ with rectangular cartesian coordinates $x_{1}, x_{2}, \cdots, x_{2 n-1}$ by the equations

$$
\begin{aligned}
& x_{2 i-1}=a_{i} e^{y_{n}} \cos \left(y_{i} / a_{i}\right) \text {, } \\
& x_{2 i}=a_{i} e^{y_{n}} \sin \left(y_{i} / a_{i}\right), \quad 1 \leqq i \leqq n-1, \\
& x_{2 n-1}=\int_{0}^{y_{n}}\left(1-e^{2 u}\right)^{1 / 2} d u \text {. }
\end{aligned}
$$

We find that the submanifold metric on $D$ is of constant negative curvature; however $D$ is not complete in this metric. 
In $\S 3$ of this paper we prove that one of the main steps in the proof of Hilbert's theorem, the construction of a global coordinate system whose coordinate vectors are unit-length asymptotic vectors, can be generalized to the $n$-dimensional context. Our treatment is based upon a theorem of Cartan, a proof of which is given in $\S 1$. Section 2 is devoted to the local properties of space forms isometrically immersed in space forms, and includes a rigidity theorem for minimal submanifolds of constant curvature.

Unless otherwise stated all manifolds are connected and $C^{\infty}$.

1. Exteriorly orthogonal symmetric bilinear forms, Let $V$ be an $n$-dimensional real vector space and let $\Phi^{1}, \Phi^{2}, \cdots, \Phi^{n}$ be $n$ symmetric bilinear forms on $V$. We say that $\Phi^{1}, \Phi^{2}, \cdots, \Phi^{n}$ are exteriorly orthogonal if

$$
\sum_{\lambda=1}^{n}\left[\Phi^{\lambda}(X, Y) \Phi^{\lambda}(Z, W)-\Phi^{\lambda}(X, W) \Phi^{\lambda}(Z, Y)\right]=0
$$

for $X, Y, Z, W \in V$.

Theorem 1. (Élie Cartan [3]). Suppose that $\Phi^{1}, \Phi^{2}, \cdots, \Phi^{n}$ are $n$ exteriorly orthogonal symmetric bilinear forms on an n-dimensional real vector space $V$ with the following property: if $X$ is a vector in $V$ such that $\Phi^{\lambda}(X, Y)=0$ for $1 \leqq \lambda \leqq n$ and for all $Y \in V$, then $X=0$. Then there exists a real orthogonal matrix $\left(a_{*}^{i}\right)$ and $n$ linear functionals $\varphi^{1}, \varphi^{2}, \cdots, \varphi^{n}$ such that

$$
\Phi^{\lambda}=\sum_{\mu} a_{\mu}^{\lambda} \varphi^{\mu} \otimes \Phi^{\mu}, \quad 1 \leqq \lambda \leqq n .
$$

It follows that $\Phi^{1}, \Phi^{2}, \cdots, \Phi^{n}$ are simultaneously diagonalized with respect to the basis dual to $\left\{\varphi^{1}, \varphi^{2}, \cdots, \varphi^{n}\right\}$. Theorem 1 is trivial when $n=1$ and when $n=2$ it is a consequence of the following wellknown fact: two symmetric bilinear forms, one of which is positive definite, can be simultaneously diagonalized.

We will find it convenient to regard $\Phi^{\lambda}$ as a linear transformation from $V$ to the dual space $V^{*}$ so that it induces a linear map

$$
\Phi^{\lambda} \wedge \Phi^{\lambda}: V \wedge V \rightarrow V^{*} \wedge V^{*} .
$$

Then $\Phi^{\lambda} \wedge \Phi^{\lambda}=0$ if and only if $\Phi^{\lambda}= \pm \varphi^{\lambda} \otimes \varphi^{\lambda}$ for some linear functional $\varphi^{\lambda}$. We can now restate Theorem 1 as follows: Suppose that $\Phi^{1}, \Phi^{2}, \cdots, \Phi^{n}$ are linear transformations from an $n$-dimensional real vector space to its dual such that $\left[\Phi^{\lambda}(X)\right](Y)=\left[\Phi^{\lambda}(Y)\right](X)$. If

$$
\bigcap_{\lambda} \operatorname{ker}\left(\Phi^{\lambda}\right)=(0) \text { and } \sum_{\lambda} \Phi^{\lambda} \wedge \Phi^{\lambda}=0,
$$

then there exists a real orthogonal matrix $\left(\alpha_{\mu}^{\lambda}\right)$ such that if 


$$
\Psi^{\lambda}=\sum_{\mu} a_{\mu}^{\lambda} \Phi^{\mu} \text {, then } \Psi^{\lambda} \wedge \Psi^{\lambda}=0 \text { for } 1 \leqq \lambda \leqq n .
$$

The first step in the proof of Theorem 1 consists of showing that there exists a vector $X$ in $V$ such that $\Phi^{1}(X), \Phi^{2}(X), \cdots, \Phi^{n}(X)$ are linearly independent. We prove this by contradiction. If $X \in V$, let $U^{*}(X)$ be the subspace of $V^{*}$ generated by $\left\{\Phi^{\lambda}(X): 1 \leqq \lambda \leqq n\right\}$ and let $p$ be the maximum dimension of $U^{*}(X)$ for $X \in V$. We assume that $p<n$. If $M$ is a vector for which the maximum dimension $p$ is attained, we can assume without loss of generality that

$$
\Phi^{1}(M), \Phi^{2}(M), \cdots, \Phi^{p}(M)
$$

are linearly independent, and $\Phi^{p+1}(M)=\cdots=\Phi^{n}(M)=0$. If $Y$ is any other vector in $V$, then

$$
\sum_{\alpha=1}^{p} \Phi^{\alpha}(M) \wedge \Phi^{\alpha}(Y)=0,
$$

so that by Cartan's lemma there exists a $p \times p$ symmetric matrix $\left(c_{\beta}^{\alpha}\right)$ such that

$$
\Phi^{\alpha}(Y)=\sum_{\beta=1}^{p} c_{\beta}^{\alpha} \Phi^{\beta}(M), \quad 1 \leqq \alpha \leqq p .
$$

If we let $W^{*}$ be the subspace of $V^{*}$ generated by

$$
\left\{\Phi^{\alpha}(X): X \in V, 1 \leqq \alpha \leqq p\right\},
$$

then (1) shows that $W^{*}$ is exactly $p$-dimensional. Since $p<n$ there exists a nonzero vector $Z$ in $V$ which is annihilated by $W^{*}$. But by hypothesis there exists $\lambda, 1 \leqq \lambda \leqq n$, and a vector $N \in V$ such that $\Phi^{\lambda}(Z, N) \neq 0$. Since $Z$ is annihilated by $W^{*}, \lambda \geqq p+1$. If $\varepsilon>0$ is sufficiently small, $\left\{\Phi^{\alpha}[(\cos \varepsilon) M+(\sin \varepsilon) N] 1 \leqq \alpha \leqq p\right\}$ will generate $W^{*}$ and $\Phi^{\lambda}[(\cos \varepsilon) M+(\sin \varepsilon) N]$ will be outside of $W^{*}$. Hence

$$
U^{*}[(\cos \varepsilon) M+(\sin \varepsilon) N]
$$

is at least $(p+1)$-dimensional; this contradicts the definition of $p$, and the first step is established.

Let $\left\{v_{1}, v_{2}, \cdots, v_{n}\right\}$ be a basis for $V$ such that

$$
\Phi^{1}\left(v_{1}\right), \Phi^{2}\left(v_{1}\right), \cdots, \Phi^{n}\left(v_{1}\right)
$$

are linearly independent. Then we can apply Cartan's lemma to the equation

$$
\sum_{\lambda} \Phi^{\lambda}\left(v_{1}\right) \wedge \Phi^{\lambda}\left(v_{i}\right)=0
$$

and conclude that there exists a symmetric matrix $C(i)=\left(c(i)_{\mu}^{i}\right)$ such that 


$$
\Phi^{\lambda}\left(v_{i}\right)=\sum_{\mu} c(i)_{\mu}^{\lambda} \Phi^{\mu}\left(v_{1}\right), \quad 1 \leqq \lambda \leqq n .
$$

(Notice that $C(1)$ is the identity matrix.) We next observe that it follows from the equation

$$
\sum_{i} \Phi^{\lambda}\left(v_{i}\right) \wedge \Phi^{\lambda}\left(v_{j}\right)=0
$$

that the matrices $C(i)$ and $C(j)$ commute with each other. By a well-known theorem from linear algebra there exists an orthogonal matrix $A=\left(a_{\mu}^{\lambda}\right)$ such that $A[C(i)]\left[{ }^{t} A\right]$ is diagonal for $1 \leqq i \leqq n$. If we let $\Psi^{\lambda}=\sum_{\mu} a_{\mu}^{\lambda} \Phi^{\mu}$ then $\Psi^{\lambda}\left(v_{i}\right)$ is a constant multiple of $\Psi^{\lambda}\left(v_{1}\right)$ for $1 \leqq i \leqq n$, so that

$$
\Psi^{\lambda}\left(v_{i}\right) \wedge \Psi^{\lambda}\left(v_{j}\right)=0, \quad 1 \leqq i, j, \lambda \leqq n .
$$

It follows that $\Psi^{\lambda} \wedge \Psi^{\lambda}=0,1 \leqq \lambda \leqq n$, and Theorem 1 is proven.

An examination of the above proof shows that $\Psi^{1}, \Psi^{2}, \cdots, \Psi^{n}$ are uniquely determined up to a permutation. Hence the linear functionals $\varphi^{1}, \varphi^{2}, \cdots, \varphi^{n}$ are uniquely determined up to changes of sign and a possible permutation.

2. Submanifolds of constant curvature: local theory. In the rest of this paper, our setup will be as follows: we will let $M$ be an $n$-dimensional riemannian manifold of constant curvature $k$ isometrically immersed in a $(2 n-1)$-dimensional riemannian manifold $N$ of constant curvature $K$. We will use the following conventions on ranges of indices:

$$
1 \leqq i, j, k, l \leqq n, \quad n+1 \leqq \lambda, u \leqq 2 n-1, \quad 1 \leqq A, B, C \leqq 2 n-1 .
$$

Let $e_{1}, e_{2}, \cdots, e_{2 n-1}$ be a moving oriented orthonormal frame on an open set $U$ in $N$, chosen so that at points of a suitable open subset $V$ of the submanifold $M$ the first $n$ frame vectors are tangent to $M$. Let $\theta^{1}, \theta^{2}, \cdots, \theta^{2 n-1}$ be the dual orthonormal coframe. A fundamental theorem of riemannian geometry states that there exists a unique collection of 1-forms $\theta_{B}^{A}$ on $U$ which satisfy the structure equation

$$
d \theta^{A}=-\sum_{B} \theta_{B}^{A} \wedge \theta^{B}, \theta_{B}^{A}=-\theta_{A}^{B}
$$

The fact that $N$ has constant curvature $K$ is expressed by the equation

$$
d \theta_{B}^{A}=-\sum_{C} \theta_{C}^{A} \wedge \theta_{B}^{C}+K \theta^{A} \wedge \theta^{B} .
$$

If we restrict these equations to the open subset $V$ of $M$ and make use of the fact that $\theta^{2}=0$ on $V$, we obtain from (2) the equations 


$$
d \theta^{i}=-\sum_{k} \theta_{k}^{i} \wedge \theta^{k}, 0=-\sum_{k} \theta_{k}^{\lambda} \wedge \theta^{k}
$$

The second of these implies via Cartan's lemma that

$$
\theta_{i}^{\lambda}=\sum_{j} b_{i j}^{\lambda} \theta^{j}, b_{i j}^{\lambda}=b_{j i}^{\lambda},
$$

where the $b_{i j}^{\lambda}$ 's are differentiable functions on $V$ called the components of the second fundamental forms. From equation (3) we obtain the equation

$$
d \theta_{j}^{i}=-\sum_{k} \theta_{k}^{i} \wedge \theta_{j}^{k}-\sum_{\lambda} \theta_{\lambda}^{i} \wedge \theta_{j}^{\lambda}+K \theta^{i} \wedge \theta^{j} .
$$

Since $M$ is of constant curvature $k$

$$
-\sum_{\lambda} \theta_{\lambda}^{i} \wedge \theta_{j}^{\lambda}=(k-K) \theta^{i} \wedge \theta^{j},
$$

or equivalently

$$
\sum_{\lambda}\left(b_{i j}^{\lambda} b_{k l}^{\lambda}-b_{i l}^{\lambda} b_{k j}^{\lambda}\right)=(k-K)\left(\delta_{i j} \delta_{k l}-\delta_{i l} \delta_{k j}\right),
$$

where $\delta_{i j}$ is the usual Kronecker delta.

Assume now that $k<K$. Equation (6) then states that the second fundamental forms $\Phi^{\lambda}=\sum_{i} \theta_{i}^{\lambda} \otimes \theta^{i}$ and the symmetric bilinear form

$$
\Psi=\sqrt{K-k}\left(\sum_{i} \theta^{i} \otimes \theta^{i}\right)
$$

are exteriorly orthogonal, and Theorem 1 implies that they can be simultaneously diagonalized by a basis for the tangent space to $M$. Since the basis diagonalizes $\Psi$ it can be chosen to be orthonormal, and hence we can assume that the moving frame $e_{1}, e_{2}, \cdots, e_{2 n-1}$ chosen in the preceding paragraphs satisfies the equations $b_{i j}^{\lambda}=0$ for $i \neq j$. In view of the remark at the end of $\S 1$, any two diagonalizing orthonormal bases differ at most by changes of sign and a possible permutation. Hence if $M$ is simply connected we can choose a global moving frame $e_{1}, e_{2}, \cdots, e_{n}$ on $M$ which diagonalizes the second fundamental forms. In particular, the universal covering space of $M$ is parallelizable.

In terms of the diagonalizing moving frame, equation (6) takes the simpler form

$$
\sum_{i} b_{i i}^{\lambda} b_{j j}^{\lambda}=(k-K), \quad i \neq j
$$

We claim that it follows from this equation that there exist unique positive functions $x_{1}, x_{2}, \cdots, x_{n}$ such that

$$
\sum_{i} b_{i i}^{\lambda} x_{i}^{2}=0 \text { and } \sum_{i} x_{i}^{2}=1 \text {. }
$$


Indeed, such functions need to satisfy the equation

$$
0=\sum_{i, \lambda} b_{j j}^{\lambda} b_{i i}^{\lambda} x_{i}^{2}=\sum_{\lambda} b_{j j}^{\lambda} b_{j j}^{\lambda} x_{j}^{2}-(K-k)\left(1-x_{j}^{2}\right),
$$

from which it follows that

$$
\sum_{i} b_{i i}^{\lambda} b_{i i}^{\lambda}=(K-k)\left(1-x_{i}^{2}\right) / x_{i}^{2} .
$$

We can solve for $x_{i}$ to obtain the expression

$$
x_{i}=\left[\left(\sum_{\lambda} b_{i i}^{\lambda} b_{i i}^{\lambda}\right) /(K-k)+1\right]^{-1 / 2},
$$

and check that the functions defined by this equation satisfy equations (8). A slight modification of this argument shows that any $n-1$ of the "principal normal curvature vectors" $\sum_{\lambda} b_{i i}^{\lambda} e_{\lambda}$ are linearly independent.

A restatement of what we proved in the preceding paragraph is that there exist exactly $2^{n}$ unit-length vectors on which all the second fundamental forms vanish simultaneously. They are all of the form

$$
\pm x_{1} e_{1} \pm x_{2} e_{2} \pm \cdots \pm x_{n} e_{n}
$$

where the signs can be chosen in $2^{n}$ ways, and they are called asymptotic vectors.

We remark that the normal bundle of $M$ in $N$ has zero curvature because the curvature forms of the normal bundle are $-\sum_{i} \theta_{i}^{\lambda} \wedge \theta_{\mu}^{i}$ and both $\theta_{i}^{\lambda}$ and $\theta_{\mu}^{i}$ are multiples of $\theta^{i}$. Hence without loss of generality we will assume that $e_{n+1}, \cdots, e_{2 n-1}$ have been chosen so that $\theta_{\mu}^{\lambda}=0$.

Our next objective is to find an expression for the differential 1-forms $\theta_{j}^{i}$ in terms of the functions $x_{i}$. For this purpose we will use the tensor $b_{i j k}^{\lambda}$ defined by the following equation

$$
d b_{i j}^{\lambda}+\sum_{\mu} b_{i j}^{\mu} \theta_{\mu}^{\lambda}-\sum_{k} b_{k j}^{\lambda} \theta_{i}^{k}-\sum_{k} b_{i k}^{\lambda} \theta_{j}^{k}=\sum_{k} b_{i j k}^{\lambda} \theta^{k} .
$$

The exterior derivative of equation (5) shows that the tensor $b_{i j k}^{i}$ is symmetric in its lower indices. If we make use of the facts that $b_{i j}^{\lambda}=0$ for $\mathrm{i} \neq j$ and $\theta_{\mu}^{\lambda}=0$, we can simplify (12) and obtain the equations

$$
\begin{gathered}
d b_{i i}^{\lambda}=\sum_{k} b_{i i k}^{\lambda} \theta^{k}, \\
\left(b_{j j}^{\lambda}-b_{i i}^{\lambda}\right) \theta_{j}^{i}=\sum_{k} b_{i j k}^{\lambda} \theta^{k}, \quad \mathrm{i} \neq j .
\end{gathered}
$$

If we choose $e_{n+1}$ at a point $x \in M$ so that $b_{11}^{n+2}(x)=\cdots=b_{11}^{2 n-1}(x)=0$, then it follows from equation (7) that 


$$
b_{i i}^{n+1}(x)=(k-K) / b_{11}^{n+1}(x) .
$$

Equation (14) therefore implies that $b_{i j k}^{n+1}(x)=0$ for $i, j, 1$ distinct. It follows that $b_{i j k}^{n+1}(x)=0$ for $i, j, k$ distinct, and since the principal normal curvature vectors span the normal space, $b_{i j k}^{\lambda}(x)=0$ for $i, j, k$ distinct. Since $x$ is arbitrary, equation (14) now becomes

$$
\left(b_{j j}^{\lambda}-b_{i i}^{\lambda}\right) \theta_{j}^{i}=b_{i j i}^{\lambda} \theta^{i}+b_{i j j}^{\lambda} \theta^{j}, \quad i \neq j .
$$

We multiply this last equation by $b_{i i}^{\lambda}$ and sum with respect to $\lambda$ to conclude that

$$
\left(k-K-\sum_{\lambda} b_{i i}^{\lambda} b_{i i}^{\lambda}\right) \theta_{j}^{i}=\sum_{\lambda} b_{i i}^{\lambda} b_{i i j}^{\lambda} \theta^{i}+\sum_{\lambda} b_{i i}^{\lambda} b_{j j \imath}^{\lambda} \theta^{j}, \quad i \neq j .
$$

We now need to use the following fact which is a consequence of (9):

$$
2 \sum_{i} b_{i i}^{\lambda} b_{i i j}^{\lambda}=(K-k) e_{j}\left[\left(1-x_{i}^{2}\right) / x_{i}^{2}\right] \text {. }
$$

We can use this to derive the following equation for the 1 -forms $\theta_{j}^{i}$ :

$$
\theta_{\jmath}^{i}=\left(1 / x_{i}\right) e_{j}\left(x_{i}\right) \theta^{i}+\text { (something) } \theta^{j} .
$$

Using skew-symmetry we conclude that

$$
\theta_{\jmath}^{i}=\left(1 / x_{i}\right) e_{j}\left(x_{i}\right) \theta^{i}-\left(1 / x_{j}\right) e_{i}\left(x_{j}\right) \theta^{j} .
$$

As an application of these ideas we prove the following theorem closely related to recent work of do Carmo and Wallach [2]:

THeOREM 2. Let $M$ be a connected n-dimensional riemannian manifold of constant curvature $k$ isometrically and minimally immersed in a simply connected $(2 n-1)$-dimensional riemannian manifold $N$ of constant curvature $K$. Then either $M$ is totally geodesic or it is flat. In the flat case it is immersed as a piece of the n-dimensional Clifford torus in the $(2 n-1)$-sphere.

The proof is local. The fact that the immersion is minimal is expressed by the equation

$$
\sum_{i} b_{i i}^{\lambda}=0
$$

which tcgether with equation (6) implies that

$$
\sum_{i, \lambda} b_{i j}^{\lambda} b_{i k}^{i}=(n-1)(K-k) \delta_{j k} .
$$

Hence $k \leqq K$ and if $k=K$ then the submanifold $M$ is totally geodesic. Therefore we assume without loss of generality that $k<K$.

In the case where $k<K$ we will actually prove a little more 
than the theorem states: if the hypothesis that $M$ be minimal is replaced by the weaker condition that its mean curvature vector be parallel, it still follows that $M$ is flat.

Since the normal moving frame vectors are parallel, the mean curvature vector is parallel if and only if there exist constants $c^{\lambda}$ such that

$$
\sum_{i} b_{i i}^{2}=c^{\lambda}
$$

On the other hand, equations (13) and (7) imply that

$$
\sum_{i} b_{i i j}^{\lambda}=0 \text {, and } \sum_{\lambda} b_{i i j}^{\lambda} b_{k k}^{\lambda}=-\sum_{\lambda} b_{i i}^{\lambda} b_{k k j}^{\lambda} \text { if } i \neq k \text {. }
$$

Hence we conclude that

$$
\begin{aligned}
\sum_{i, \lambda} b_{i i,}^{\lambda} b_{i i}^{\lambda} & =-\sum_{\substack{\lambda \\
k \neq i}} b_{k k_{j}}^{\lambda} b_{i i}^{\lambda}=\sum_{\substack{\lambda \\
k \neq i}} b_{k k k}^{\lambda} b_{i i j}^{\lambda} \\
& =\sum_{i, \lambda} c^{\lambda} b_{i i j}^{\lambda}-\sum_{i, \lambda} b_{i i}^{\lambda} b_{i i j}^{\lambda}=-\sum_{i, \lambda} b_{i i}^{\lambda} b_{i i j}^{\lambda} .
\end{aligned}
$$

It follows that $\sum_{i, \lambda} b_{i i j}^{\lambda} b_{i i}^{\lambda}=0$, and hence equation (15) implies that $e_{j}\left(x_{i}\right)=0$. Now by equation (16) the differential forms $\theta_{j}^{i}$ vanish, proving that $M$ is flat.

To finish the proof of the theorem, we notice that if $M$ is minimal the principal normal curvature vectors (i.e., the $b_{i i}^{\prime}$ 's) are determined up to a rotation of $e_{n+1}, \cdots, e_{2 n-1}$ by equations (7) and (17). Since the $b_{i i}^{\lambda}$ 's determine the $\theta_{i}^{\prime \prime}$ 's and $\theta_{j}^{i}=0=\theta_{\mu}^{\lambda}$, it follows from the classical rigidity theorem [1, p. 202] that locally there is at most one minimal flat $n$-dimensional submanifold of $N$, up to a rigid motion. Therefore $M$ must be a piece of the Clifford torus, and the theorem is proven.

3. The global existence of asymptotic coordinates. If $M$ is complete and simply connected, then any choice of signs in expression (11) determines a globally defined unit-length asymptotic vector field on $M$. If $n$ unit-length asymptotic vector fields are linearly independent at one point, they are linearly independent everywhere.

THEOREV 3. If $M$ is a complete simply connected riemannian manifold of constant curvature $k$ isometrically immersed in a $(2 n-1)$ dimensional riemannian manifold $N$ of constant curvature $K>k$, then any $n$ linearly independent unit-length asymptotic vector fields $Z_{1}, Z_{2}, \cdots, Z_{n}$ determine a global coordinate system whose coordinate vectors are the $Z_{i}$ 's.

First we establish local existence. Because of the theorem of Frobenius, it suffices to show that the Lie bracket of any two asymp- 
totic vector fields is zero. But

$$
\begin{aligned}
\theta^{i}\left(\left[x_{j} e_{j}, x_{k} e_{k}\right]\right) & =x_{j} e_{j}\left(\theta^{i}\left(x_{k} e_{k}\right)\right)-x_{k} e_{k}\left(\theta^{i}\left(x_{j} e_{j}\right)\right)-2 d \theta^{i}\left(x_{j} e_{j}, x_{k} e_{k}\right) \\
& =x_{j} e_{j}\left(\theta^{i}\left(x_{k} e_{k}\right)\right)-x_{k} e_{k}\left(\theta^{i}\left(x_{j} e_{j}\right)\right)+2 \sum_{l} \theta_{l}^{i} \wedge \theta^{l}\left(x_{j} e_{j}, x_{k} e_{k}\right) \\
& =\delta_{i k} x_{j} e_{j}\left(x_{k}\right)-\delta_{i j} x_{k} e_{k}\left(x_{j}\right)+\delta_{i j} x_{k} e_{k}\left(x_{j}\right)-\delta_{i k} x_{j} e_{j}\left(x_{k}\right) \\
& =0 .
\end{aligned}
$$

In this derivation we have used equations (4) and (16). Since the asymptotic vectors are sums of $\pm x_{i} e_{i}$, local existence is proven.

To prove global existence, we let $\varphi_{i}(x, t), x \in M, t \in \boldsymbol{R}$ be the one-parameter group of transformations corresponding to $Z_{i}$. Since $Z_{i}$ is a vector field of unit length, it follows from the theory of ordinary differential equations $[4, \mathrm{p} .15]$ that $\varphi_{i}(x, t)$ is defined for all values of $x$ and $t$. Let $x_{0}$ be a fixed point in $M$ and define a function $F$ : $\boldsymbol{R}^{n} \rightarrow M$ by

$$
F\left(t_{1}, t_{2}, \cdots, t_{n}\right)=\varphi_{n}\left(\varphi_{n-1}\left(\cdots \varphi_{2}\left(\varphi_{1}\left(x_{0}, t_{1}\right), t_{2}\right), \cdots\right), t_{n}\right) .
$$

Since the Lie bracket $\left[Z_{i}, Z_{j}\right]$ vanishes, the one-parameter groups $\varphi_{i}$ and $\varphi_{j}$ commute. Using this fact we can verify the following equation:

$$
F\left(s_{1}+t_{1}, \cdots, s_{n}+t_{n}\right)=\varphi_{n}\left(\varphi_{n-1}\left(\cdots \varphi_{1}\left(F\left(s_{1}, \cdots, s_{n}\right), t_{1}\right), \cdots\right), t_{n}\right) .
$$

We claim that $F$ is a covering map. Let $x$ be a point in the manifold $M$ and let $U_{x}$ be an open neighborhood of $x$ on which local asymptotic coordinates $z_{1}, z_{2}, \cdots, z_{n}$ exist, and we can assume that $z_{1}(x)=z_{2}(x)=\cdots=z_{n}(x)=0$. For $\delta>0$, let

$$
B_{\hat{o}}(x)=\left\{y \in U_{x}:\left|z_{i}(y)\right|<\delta\right\}
$$

and choose $\varepsilon$ so small that $\left(z_{1}, z_{2}, \cdots, z_{n}\right)$ give a diffeomorphism from $B_{2 \varepsilon}(x)$ onto an open ball of radius $2 \varepsilon$ in $\boldsymbol{R}^{n}$. Let $\widetilde{x}_{\alpha}, \alpha \in A$, be the points in $F^{-1}(x)$, and let $B_{\delta}\left(\widetilde{x}_{\alpha}\right)$ denote the open ball of radius $\delta$ around $x_{\alpha}$. To show that $F$ is a covering map, it suffices to check the following facts:

1. $F \mid B_{2 \varepsilon}\left(\widetilde{x}_{\alpha}\right)$ is a diffeomorphism from $B_{2 \varepsilon}\left(\widetilde{x}_{\alpha}\right)$ onto $B_{2 \varepsilon}(x)$ for $\alpha \in A$.

2. $B_{\varepsilon}\left(\widetilde{x}_{\alpha}\right) \cap B_{\varepsilon}\left(\widetilde{x}_{\beta}\right)=\phi$ if $\widetilde{x}_{\alpha} \neq \widetilde{x}_{\beta}$.

3. $\widetilde{y} \in F^{-1}\left(B_{\varepsilon}(x)\right) \Longrightarrow \widetilde{y} \in B_{\varepsilon}\left(\widetilde{x}_{\alpha}\right)$ for some $\alpha \in A$.

To prove 1 , we need only check that the local asymptotic coordinates define an inverse to $F \mid B_{2 \varepsilon}\left(\widetilde{x}_{\alpha}\right)$ using equation (18). 2 follows from 1 , and 3 follows from the fact that $\widetilde{y}-\left(z_{1}(F(\widetilde{y})), \cdots, z_{n}(F(\widetilde{y}))\right)$ goes to $x$ under $F$.

Thus $F$ is a covering map, and since $M$ is simply connected it is a diffeomorphism. Therefore $F$ defines a global coordinate system whose coordinate vectors are the $Z_{i}$ 's and Theorem 3 is proven. 
A straightforward modification of the above proof establishes the existence of "principal coordinates" whose coordinate vectors are $x_{1} e_{1}, x_{2} e_{2}, \cdots, x_{n} e_{n}$.

Since $\boldsymbol{R}^{n}$ is not a covering space for the $n$-sphere when $n>1$, we obtain the positive curvature analogue of our conjecture:

Corollary. A complete n-dimensional riemannian manifold of constant positive curvature $k$ cannot be isometrically immersed in $a(2 n-1)$-sphere of constant curvature $K>k$.

The corresponding local assertion is false, as Cartan proved in [3]. An $n$-sphere of constant curvature can be isometrically immersed in a $(2 n+1)$-sphere of constant curvature by first embedding it in $E^{n+1}$ in the usual fashion, and then immersing $E^{n+1}$ in the $(2 n+1)$ sphere as a flat torus.

If $M$ is a complete simply connected space form as in Theorem 3 , we will use the term "asymptotic surface" to denote a complete two-dimensional submanifold generated by two unit-length asymptotic vector fields. Every asymptotic surface possesses a global Tchebychef net ([7], p. 198) and it follows from the formula of Hazzidakkis that the integral of the Gaussian curvature over any parallelogram of the Tchebychef net is bounded in absolute value by $2 \pi$.

\section{REFERENCES}

1. R. L. Bishop and R. J. Crittenden, Geometry of manifolds, Academic Press, New York, 1964.

2. M. P. do Carmo and N. J. Wallach, Minimal immersions of spheres into spheres, Ann. of Math., 93 (1971), 43-62.

3. É. Cartan, Sur les variétés de courbure constante d'un espace euclidien ou noneuclidien, Bull. Soc. Math. France, 47 (1919), 125-160 and 48 (1920), 132-208.

4. E. Coddington and N. Levinson, Theory of ordinary differential equations, McGrawHill, New York, 1955.

5. D. Hilbert, Über Flächen von konstanter Gausscher Krümmung, Trans. Amer. Math. Soc., 2 (1901), 87-99.

6. S. Kobayashi and K. Nomizu, Foundations of differential geometry, 2 vols., Interscience, New York, 1969.

7. J. J. Stoker, Differential Geometry, Wiley-Interscience, New York, 1969.

Received October 30, 1970. This paper is a revised version of part of the author's dissertation written under the direction of Professor Kobayashi at the University of California, Berkeley. The author wishes to express his gratitude to Professors do Carmo and Kobayashi for their encouragement and helpful suggestions.

UNIVERSity OF CALIFORNIA, SANTA BARBARA 


\section{PACIFIC JOURNAL OF MATHEMATICS}

\section{EDITORS}

H. SAMELSON

Stanford University

Stanford, California 94305

C. R. HOBBY

University of Washington

Seattle, Washington 98105
J. DugundJI

Department of Mathematics

University of Southern California

Los Angeles, California 90007

RICHARD ARENS

University of California

Los Angeles, California 90024

\section{ASSOCIATE EDITORS}

E. F. BeCKENBACH

B. H. NeumanN

F. WOLF

K. YosHIDA

\section{SUPPORTING INSTITUTIONS}

UNIVERSITY OF BRITISH COLUMBIA

CALIFORNIA INSTITUTE OF TECHNOLOGY

UNIVERSITY OF CALIFORNIA

MONTANA STATE UNIVERSITY

UNIVERSITY OF NEVADA

NEW MEXICO STATE UNIVERSITY

OREGON STATE UNIVERSITY

UNIVERSITY OF OREGON

OSARA UNIVERSITY
UNIVERSITY OF SOUTHERN CALIFORNIA STANFORD UNIVERSITY

UNIVERSITY OF TOKYO

UNIVERSITY OF UTAH

WASHINGTON STATE UNIVERSITY

UNIVERSITY OF WASHINGTON

AMERICAN MATHEMATICAL SOCIETY

NAVAL WEAPONS CENTER

Printed in Japan by International Academic Printing Co., Ltd., Tokyo, Japan 


\section{Pacific Journal of Mathematics}

\section{Vol. 40, No. $1 \quad$ September, 1972}

Alex Bacopoulos and Athanassios G. Kartsatos, On polynomials

approximating the solutions of nonlinear differential equations........

Monte Boisen and Max Dean Larsen, Prüfer and valuation rings with zero

divisors ..........................................

James J. Bowe, Neat homomorphisms

David W. Boyd and Hershy Kisilevsky, The Diophantine equation

$$
u(u+1)(u+2)(u+3)=v(v+1)(v+2) \ldots \ldots \ldots \ldots \ldots \ldots \ldots
$$

George Ulrich Brauer, Summability and Fourier analysis ...............

Robin B. S. Brooks, On removing coincidences of two maps when only one,

rather than both, of them may be deformed by a homotopy ............

Frank Castagna and Geert Caleb Ernst Prins, Every generalized Petersen

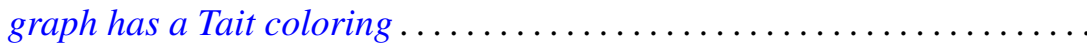

Micheal Neal Dyer, Rational homology and Whitehead products ..........

John Fuelberth and Mark Lawrence Teply, The singular submodule of a

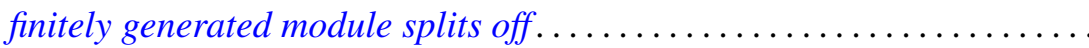

Robert Gold, $\Gamma$-extensions of imaginary quadratic fields ............ 83

Myron Goldberg and John W. Moon, Cycles in k-strong tournaments.......

Darald Joe Hartfiel and J. W. Spellmann, Diagonal similarity of irreducible

matrices to row stochastic matrices...............

Wayland M. Hubbart, Some results on blocks over local fields ..

Alan Loeb Kostinsky, Projective lattices and bounded homomorphisms....

Kenneth O. Leland, Maximum modulus theorems for algebras of operator

valued functions ...

Jerome Irving Malitz and William Nelson Reinhardt, Maximal models in the

language with quantifier "there exist uncountably many" ..

John Douglas Moore, Isometric immersions of space forms in space

forms.

Ronald C. Mullin and Ralph Gordon Stanton, A map-theoretic approach to

Davenport-Schinzel sequences ....................

Chull Park, On Fredholm transformations in Yeh-Wiener space. .

Stanley Poreda, Complex Chebyshev alterations ..............

Ray C. Shiflett, Extreme Markov operators and the orbits of Ryff. ...

Robert L. Snider, Lattices of radicals .....................

Ralph Richard Summerhill, Unknotting cones in the topological

category ................................

Charles Irvin Vinsonhaler, A note on two generalizations of $\mathrm{QF}-3 \ldots \ldots 229$

William Patterson Wardlaw, Defining relations for certain integrally

parameterized Chevalley groups...................

William Jennings Wickless, Abelian groups which admit only nilpotent

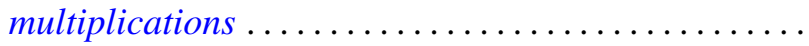

